298-300) that future developments in genetics may lead to tailored treatments for psychiatric patients. Currently in addiction services, these technologies are not yet generally in use. However, recent developments suggest they may soon be available to patients

Like antipsychotic drugs, nicotine is metabolised by a cytochrome enzyme complex (CYP 2A6). In past years, much attention has focused on the effect of functional variants of the CYP 2A6 gene on smoking status (Pianezza et al, 1998). More recent studies have also highlighted the potential of applying pharmacogenetics in clinical addiction services; women receiving nicotine replacement therapy (NRT) possessing a variant of dopamine receptor 2 gene (DRD2) were shown to have significantly different success rates with NRT depending on their DRD2 genotype (Yudkin et al, 2004).

This and other findings (Lerman et al, 2002) raise the issue of screening smokers with the intention of informing them which treatments they are most likely to benefit from. With rapid advances in genomic information and high throughput genotype screening techniques, more relevant functional genomic information is becoming available. Hodgson et al correctly infer that a thorough development phase is needed before this approach can be translated into widespread clinical applications.

In addition it will be important to protect patients against possible premature exposure to private genetic screening and advisory services using preliminary genetic findings which may not be substantiated through rigorous replication studies. With the realistic possibility of commercial involvement in gene based diagnostics, patients may be exposed to marketing strategies offering tailored smoking cessation therapies, based on preliminary/incomplete study results.

LERMAN, C., SHIELDS, P. G., WILEYTO, E. P. et al (2002) Pharmacogenetic investigation of smoking cessation treatment. Pharmacogenetics, 12, 627634.

PIANEZZA, M. L., SELLERS, E. M. \& TYNDALE, R. F. (1998) Nicotine metabolism defect reduces smoking. Nature, 393, 750.

YUDKIN, P. (2004) Effectiveness of nicotine patches in relation to genotype in women versus men: randomised controlled trial. BMJ, 328, 989-990.

Colin O'Gara Clinical Lecturer in the Addictions, Institute of Psychiatry and Honorary Specialist Registrar, Smoking Cessation Clinic, Maudsley Hospital, Denmark Hill, London SE5 8AF.

E-mail: c.o'gara@iop.kcl.ac.uk

\section{Why do psychiatric patients wait too long in A\&E?}

By December 2004, all patients must be discharged from an accident and emergency (A\&E) department within 4 hours of arrival (Department of Health, 2001). We sought to identify what factors contributed to the long waiting times experienced by some patients referred to psychiatry by our local $A \& E$ department.

Over a 2-month period in 2004, we identified 23 patients who breached the 4-hour target. More than half were patients who had self-harmed. Alcohol intoxication and awaiting the results of investigations following an overdose were common reasons for a delay. In more than a fifth of cases, a prolonged psychiatric assessment was required, including one Mental Health Act 1983 assessment. In $40 \%$ of cases, there was more than an hour's delay between referral and psychiatric assessment.

A rapid response is unlikely to reduce attendance to below 4 hours in all cases. If a psychiatrist can attend within 1 hour, and their assessment takes no more than another hour, then patients should be referred within 2 hours of attendance to achieve the 4-hour target. This occurred in only one-quarter of cases reviewed.

Striving to achieve rapid throughput for patients with psychiatric as well as physical problems may not always be possible or advisable (Psychiatric Bulletin, December 2003, 27, 81-82). Obtaining background information is often a crucial but time-consuming part of an assessment. Is the drive for a rapid discharge from $A \& E$, evidence of a sway towards 'fast psychiatry' (Psychiatric Bulletin, July 2004, 28, 265-266) that runs counter to good clinical care?

DEPARTMENT OF HEALTH (2001) Reforming Emergency Care: First Steps of a NewApproach. London: Department of Health.

Rina Dutta Senior House Officer Dolores Velazquez Specialist Registrar, St George's Rotation, South Thames (West) Region, London, Jim Bolton Consultant Liaison Psychiatrist, St Helier Hospital, Surrey and Honorary Senior Lecturer, St George's Hospital Medical School, ondon. 